\title{
Electron-beam-stimulated Atomic Migration Processes in Single-layer MoTe2
}

Janis Köster ${ }^{1}$, Tibor Lehnert ${ }^{1}$, Mahdi Ghorbani-Asl ${ }^{2}$, Silvan Kretschmer ${ }^{2}$, Hannu-Pekka Komsa ${ }^{3}$, Arkady Krasheninnikov $^{2}$ and Ute Kaiser ${ }^{1}$

${ }^{1}$ Ulm University, Ulm, Baden-Wurttemberg, Germany, ${ }^{2}$ Helmholtz-Zentrum Dresden-Rossendorf, Dresden, Sachsen, Germany, ${ }^{3}$ Aalto University, Aalto, Uusimaa, Finland

Low-voltage aberration-corrected transmission electron microscopy (TEM) allows in-situ imaging of atomic migration processes in two-dimensional (2D) materials. 2D transition metal dichalcogenides (TMDs) have received great attention in recent years due to their unique properties, they enable a wide range of applications in the fields of electronics, optoelectronics, sensor and quantum technologies [1]. In the latter case MoTe2, the TMD studied in this work, is a very promising candidate $[2,3,4]$.

To secure sub-Ångström resolution, high image contrast, and to avoid ballistic damage to the MoTe2 single layer during imaging, we performed our experiments using the spherical and chromatic aberrationcorrected SALVE (sub-Ångström low-voltage electron microscopy) instrument operated at $40 \mathrm{kV}[5,6$, $7,8,9]$. In earlier studies, we reported about electron-beam-induced evolution of single Te vacancies into tetravacancies exhibiting magnetic properties [10]. Here, we study in-situ the dynamics and the temporal lifetime of Te vacancies of various defect configurations in the single-layer $1 H$-MoTe2. Additionally, electron-beam-driven dynamics of defects was observed and the kinetic pathways were confirmed with the DFT calculations. The calculated results indicate that atom migration occurs due to increasing vacancy concentration. Moreover, single Te vacancy lines are found to be very preferred configurations, which can perform movements (cf. Fig 1 (a)-(c)) due to the deposited energy of the electron beam and the in-plane strain in the monolayer (cf. Fig $1 \mathrm{~d}$ )). We show that induced in-plane strain due to increased vacancy concentration has a major influence on defect dynamics and transformations in monolayer MoTe2, which for example gives rise to local phase transformations from the $2 H$ to the $1 T^{\prime}$ phase (cf. Fig. 2 (a)-(b)). These results point out that defect-associated metallic quantum dots consisting of $T^{\prime}$-phase-islands can be introduced into a semiconducting single-layer $2 \mathrm{H}$-MoTe2, by creating first single Te vacancy lines. Further electron-beam-irradiation results in a high Te-deficiency, which even can lead to the formation of inversion domains with 4|4P, 4|4E and 55|8 mirror twin boundaries.

Our results describe the behavior of $\mathrm{MoTe}_{2}$ under the $40 \mathrm{keV}$ electron beam. From point to extended defects, the trajectory of the defect evolution can be understood and is interpreted as a relaxation of the in-plane strain within the 2D hexagonal matrix. This electron-beam-induced monolayer manipulation may pave a way for optical and electrical property engineering in two-dimensional materials. 
(a)

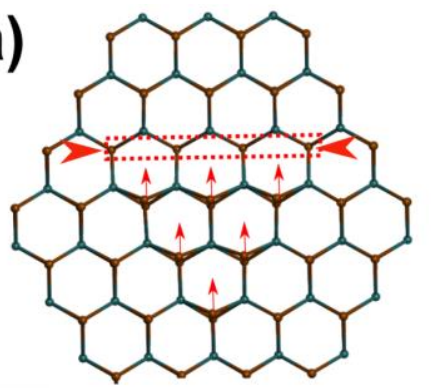

(b)

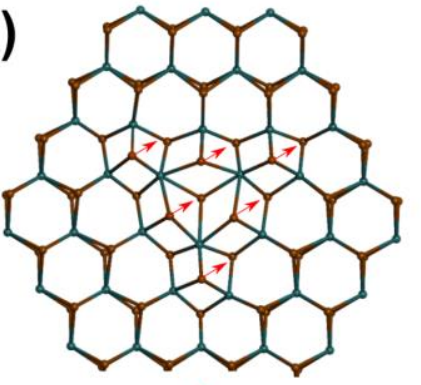

(c)

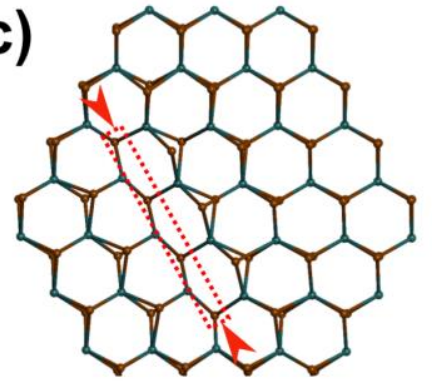

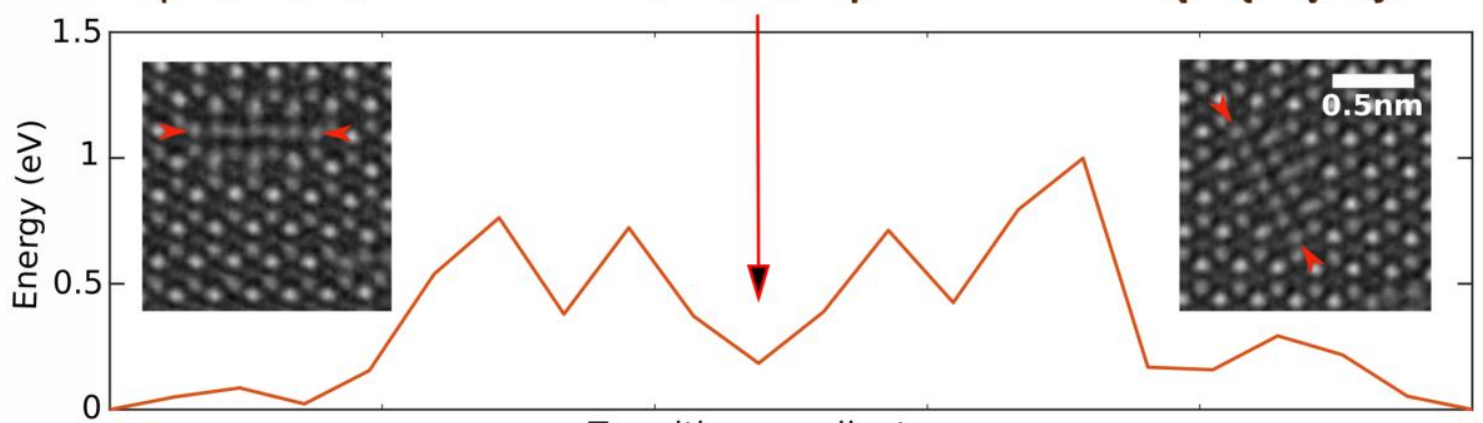
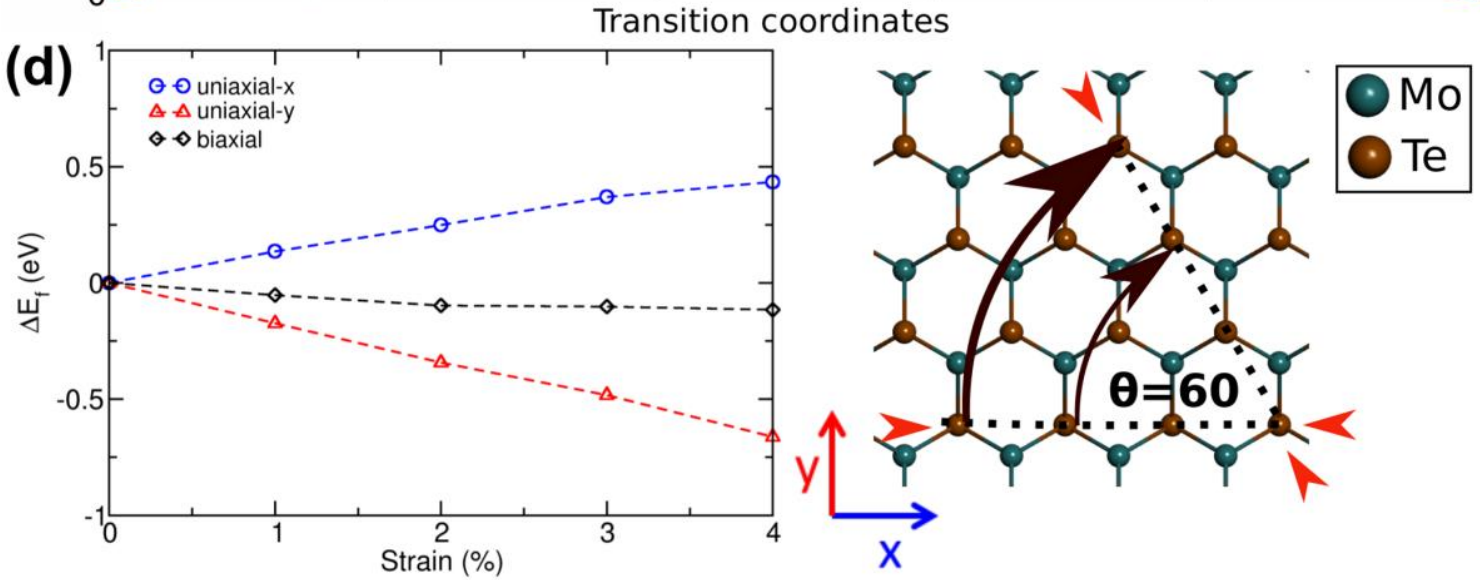

Figure 1. (a)-(c) illustrates via structure schemes a $60^{\circ}$ rotation. Corresponding $40 \mathrm{kV} \mathrm{Cc} / \mathrm{Cs}-$ corrected high-resolution SALVE images, showing a rotation in monolayer $2 \mathrm{H}-\mathrm{MoTe}$, are depicted below (a) and (c). The line defect is marked by red arrows. The orange curve shows the corresponding energy pathway. Due to fast translation, the intermediate state in (b) is not detected. In (d), a strain diagram is shown that specifies the energy gain $(\Delta \mathrm{Ef})$, which is generated by rotation of $60^{\circ}$ with different applied strain. The blue curve indicates that there is no energy gain from rotation, with strain parallel (uniaxial-x) to the single Te vacancy line. In comparison, strain perpendicular to the line defect (uniaxial-y), indicated by the red curve, gains energy and a $60^{\circ}$ rotation can be accomplished. The strain which is biaxial to the line defect has no preferred orientation (black curve). 

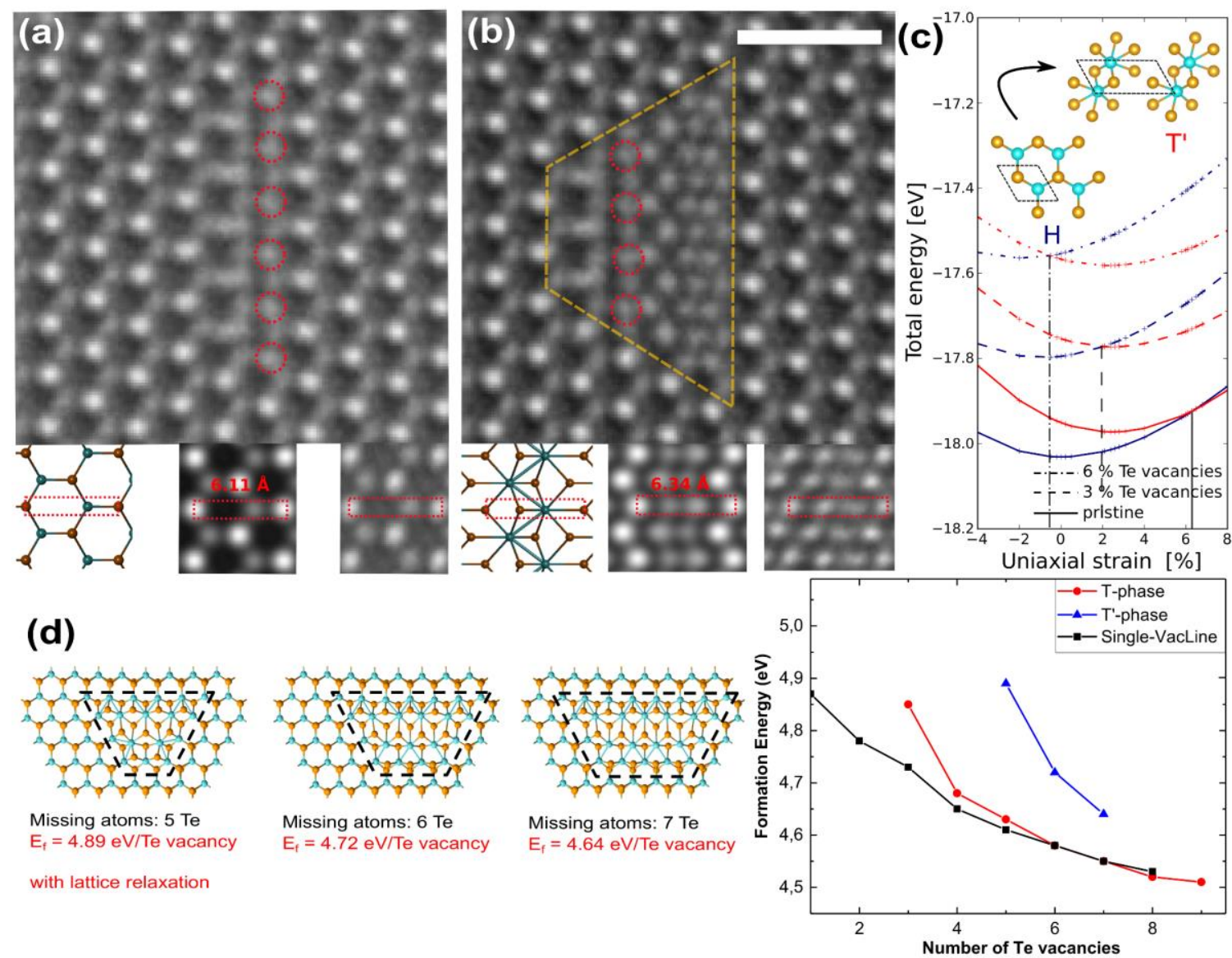

Figure 2. $40 \mathrm{kV} \mathrm{Cc/Cs-corrected} \mathrm{high-resolution} \mathrm{SALVE} \mathrm{images} \mathrm{of} \mathrm{different} \mathrm{defect} \mathrm{structures} \mathrm{in} 1 \mathrm{H}-$ MoTe2. In (a), HRTEM image of a single Te vacancy line with six missing Te atoms (indicated with red dotted rings) is shown. (a)-(b) shows the local 1T'-phase transformation. An orange trapeze in (b) highlights the area of the new 1T'-phase structure. The depictions below (a) and (b) are (from left to right) a structure simulation, an image simulation, and an experimental image of the two phases to identify the new defect structure as a localized 1T'-phase. (c) shows the total energy of the two phases depending on the applied uniaxial strain. Calculations are performed for three different vacancy concentrations. The intersection points between the blue (H-phase) and red ( $\mathrm{T}$ '-phase) curves indicate that the $\mathrm{T}$ '-phase becomes more favored with increasing vacancy concentration resulting in higher uniaxial strain. Additionally, DFT-based structure models of the T'-phase are presented in (d), and the formation energies of the T'-phase (blue) are compared in the diagram to a single Te vacancy line (black) as well as a T-phase (red). The scale bar in (b) is $1 \mathrm{~nm}$.

\section{References}

[1] Chhowalla, Manish, et al. Nature chemistry 5.4 (2013): 263

[2] Lin, Yen-Fu, et al. Advanced Materials 26.20 (2014): 3263-3269

[3] Jiang, Juan, et al. Nature communications 8 (2017): 13973

[4] Villegas, Cesar EP, and A. R. Rocha. The Journal of Physical Chemistry C 119.21 (2015): 11886-11895

[5] M. Linck et al., Phys. Rev. Lett. 2016, 117, 076101

[6] Z. Lee et al., Ultramicroscopy 2012, 112(1), 39-46

[7] H.-P. Komsa et al., Phys. Rev. Lett. 108 (2012) 196102 
[8] $\quad \underline{w w w . s a l v e-p r o j e c t . d e}$

[9] Kaiser, Ute, et al. Ultramicroscopy 111.8 (2011): 1239-1246

[10] T. Lehnert et al., ACS Appl. Nano. Mat. 2 (2019): 3262-3270

The authors acknowledge financial support by the DFG in the frame of the "SALVE" (Sub-Angström Low-Voltage Electron Microscopy) project as well as the Ministry of Science, Research and the Arts (MWK) of Baden Württemberg. 